\title{
Genetic diversity and antimicrobial resistance in Streptococcus agalactiae strains recovered from female carriers in the Bucharest area
}

\author{
Codruţa-Romaniţa Useinn,2/+, Mădălina Militaru', Violeta Cristea ${ }^{3}$, Monica Străut $^{1}$ \\ ${ }^{1}$ Molecular Epidemiology Laboratory, Cantacuzino National Institute of Research/Development for Microbiology and Immunology, \\ Bucharest, Romania ${ }^{2}$ Departament 2/Microbiology II, Carol Davila University of Medicine and Pharmacy, Bucharest, Romania \\ ${ }^{3}$ Synevo Laboratories, Judetul Ilfov, Romania
}

For the first time, we used multilocus sequence typing (MLST) to understand how Romanian group B streptococcus (GBS) strains fit into the global GBS population structure. Colonising isolates recovered from adult human females were tested for antibiotic resistance, were molecularly serotyped based on the capsular polysaccharide synthesis (cps) gene cluster and further characterised using a set of molecular markers (surface protein genes, pilus-encoded islands and mobile genetic elements inserted in the scpB-lmb intergenic region). Pulsed-field gel electrophoresis was used to complement the MLST clonal distribution pattern of selected strains. Among the 55 strains assigned to six cps types (Ia, Ib, II-V), 18 sequence types (STS) were identified by MLST. Five STs represented new entries to the MLST database. The prevalent STs were ST-1, ST-17, ST-19 and ST-28. Twenty molecular marker profiles were identified. The most common profiles ( $\mathrm{rib}+G B S i 1+P I-1, \mathrm{rib}+G B S i 1+P I-1, P I-2 b$ and alp2/3+PI-1, PI2a) were associated with the $\mathrm{cps} I I I / S T-17$ and $\mathrm{cps}$ V/ST-1 strains. A cluster of fluoroquinolone-resistant strains was detected among the cps V/ST-19 members; these strains shared alp1 and IS1548 and carried PI-1, PI-2a or both. Our results support the usefulness of implementing an integrated genotyping system at the reference laboratory level to obtain the reliable data required to make comparisons between countries.

Key words: Streptococcus agalactiae - MLST - fluoroquinolone resistance - pilus islands

Despite prophylactic perinatal antibiotic treatment, which has significantly diminished the burden of early onset disease, Streptococcus agalactiae [group B streptococcus (GBS)] remains a major infectious threat in neonates. Consequently, the development of a vaccine against GBS is a priority in health care research. Efforts to develop a vaccine capable of conferring broad coverage are also justified by the new challenges arising in GBS epidemiology, such as the diversification of the GBS serotypes (Slotved et al. 2007), a shift in serotype prevalence (Diedrick et al. 2010), capsular switching among strains (Bellais et al. 2012), a rising incidence of invasive disease in adults (Edwards \& Baker 2005) and a growing rate of reduced GBS susceptibility to antimicrobials (Castor et al. 2008). In this context, the monitoring of circulating strains and their characteristics using laboratory surveillance is pivotal for current and future vaccine development strategies.

As knowledge about the GBS genome has accumulated, genotypic systems have been developed and improved to investigate the population structure of this organism and to distinguish differences between strains isolated from different sources. Pulsed-field gel electro-

doi: 10.1590/0074-0276140431

Financial support: MECTS (NUCLEU PN 09-22 0101)

+ Corresponding author: rusein@cantacuzino.ro

Received 6 September 2013

Accepted 29 January 2014 phoresis (PFGE) and multilocus sequence typing (MLST) systems established for GBS have proven to be sufficiently discriminatory for epidemiological studies (Fasola et al. 1993, Jones et al. 2003). MLST was subsequently associated with a public website and a $S$. agalactiae database and has become a useful method for unifying global epidemiology data on asymptomatic carriage of and infection by this pathogen. MLST revealed distinct GBS sequence types (STs) that were strongly associated with invasive disease cases. Of note, the ST-17 lineage has been associated with neonatal infection, which facilitated the development of a rapid-screening molecular assay for routine diagnostic practice (Lamy et al. 2006). Population-based surveillance studies in different regions of the world have shown that the distribution of the GBS genetic lineages varies by population, which could limit the effectiveness of a universal GBS vaccine (Marchaim et al. 2006, Bohnsack et al. 2008, Manning et al. 2009).

Few molecular epidemiology studies of $S$. agalactiae have been undertaken in our region, mainly due to the high costs and the logistical difficulties that are encountered. Consequently, the paucity of information has hindered us from discerning the distinctive genomic traits of the local isolates and understanding how these isolates fit into the global population structure of this organism. This study is the first to use MLST genotyping to investigate the clonal relationships and lineage distributions of GBS isolates recovered from female carriers residing in the Bucharest metropolitan area. In addition to MLST, antibiotic resistance and the expression of a set of molecular markers associated with the capsular poly- 
saccharide synthesis (cps) gene cluster, virulence-associated surface protein antigen genes and mobile genetic elements (MGEs) were comprehensively characterised. The distributions of pathogenicity islands (PI)-1 and PI-2 (variants PI-2a and PI-2b), which carry the genes required for the biosynthesis of pili (Telford et al. 2006), were also investigated because of the importance of pili in bacterial pathogenesis and vaccine development strategies (Lauer et al. 2005, Margarit et al. 2009).

\section{SUBJECTS, MATERIALS AND METHODS}

Bacterial strains - A total of 55 non-invasive GBS strains from the Bucharest metropolitan area were included in this study. These strains were recovered from vaginal swab specimens (1 isolate per specimen) of $55 \mathrm{ep}$ idemiologically unrelated, nonpregnant adult women and the samples were received by a large, private clinical laboratory in the community. Forty-three strains collected in 2011 were fully characterised in this study. Another set of 12 strains, collected in 2010, were only subjected to MLST analysis and pilus island-associated gene assessment, as their antimicrobial resistance, capsular genotype, surface protein encoding genes and MGEs were defined in a previous study (Usein et al. 2012). Four of these strains that were identified as ST-17 by the rapid polymerase chain reaction (PCR) assay were validated using the MLST method to rule out any genotypic discrepancy.

Template DNA - Template DNA was prepared from GBS cultures using a Chelex-based boiling procedure. GBS cells were cultured overnight on Columbia agar base (Oxoid, Basingstoke, UK) supplemented with 5\% defibrinated sheep blood. Then, the cultures were suspended in $200 \mu \mathrm{L}$ of $10 \%$ Chelex 100 resin (BioRad) dissolved in TE buffer (10 mM Tris-HCl, 1 mM EDTA, $\mathrm{pH}$ 8.0); the cultures were boiled for $10 \mathrm{~min}$ and centrifuged for $5 \mathrm{~min}$ at 13,000 rpm. After centrifugation, $100 \mu \mathrm{L}$ of the supernatant, which contained total bacterial DNA, was removed and stored at $-20^{\circ} \mathrm{C}$ until further use in the molecular assays.

Antimicrobial susceptibility - The isolates were tested for susceptibility to penicillin (10 U), erythromycin $(15 \mu \mathrm{g})$, clindamycin $(2 \mu \mathrm{g})$, tetracycline $(30 \mu \mathrm{g})$, levofloxacin $(5 \mu \mathrm{g})$ and moxifloxacin $(5 \mu \mathrm{g})$ using the disk diffusion method; the European Committee on Antimicrobial Susceptibility Testing susceptibility breakpoints were used for all of the antibiotics (Oxoid) (eucast.org/ clinical_breakpoints). Isolates sensitive to clindamycin, but resistant to erythromycin, were further tested using the double-disk diffusion or D-zone test to determine whether resistance to clindamycin could be induced and these isolates were classified as having either the inducible macrolide, lincosamide and streptogramin B $\left(\mathrm{iMLS}_{\mathrm{B}}\right.$ ) antibiotic resistance phenotype or the M phenotype. Isolates resistant to clindamycin and erythromycin were confirmed as the constitutive $\mathrm{MLS}_{\mathrm{B}}$ phenotype.

PCR-based protocols using previously described primers were performed to detect the presence of the macrolide and tetracycline-resistance genes: ermA, ermB, mefA, tet $M$, tet $O$ and tet $L$ (Usein et al. 2012).

The gyrA and $\operatorname{parC}$ gene fragments, including the quinolone resistance-determining regions (QRDRs), were PCR amplified from the GBS isolates that were resistant to levofloxacin and moxifloxacin using previously published primers (Kawamura et al. 2003). The 474 and $586 \mathrm{bp}$ amplification products of gyrA and $\operatorname{par} C$, respectively, were sequenced in both directions using the same primers. DNA sequencing reactions were performed with the Big Dye Terminator v.3.1 Cycle Sequencing kit (Applied Biosystems, Foster City, California, USA) and were analysed in an ABI 3130 sequencer (Applied Biosystems). The nucleotide and deduced protein sequences were analysed using publicly available software (BioEdit) and compared with the $S$. agalactiae A909 gyrA and parC sequences, which are available in the GenBank database (accession NC_007432.1).

Molecular typing - MLST - The internal fragments of seven housekeeping genes (adhP, pheS, atr, gln $A, s d h A$, $g l c K, t k t$ ) were amplified for MLST, according to the GBS scheme described by Jones et al. (2003). All PCR products were purified using a commercial kit (Wizard SV Gel and PCR Clean-Up System, Promega, Madison, Wisconsin, USA) according to the kit instructions and sequenced on both strands. Sequencing was performed with the ABI Prism BigDye Terminator v.3.1 Cycler Sequencing kit (Applied Biosystems). Before subsequent capillary electrophoresis on an ABI 3130 Genetic Analyzer (Applied Biosystems), the sequencing products were purified using the DyeEx 2.0 Spin kit (Qiagen GmbH, Hilden, Germany). Raw sequence traces were reviewed by visual inspection using the BioEdit software. The consensus sequences were submitted to the $S$. agalactiae database (pubmlst. org/sagalactiae/) to obtain an allelic profile and to assign a ST. The MLST data generated by the study isolates were further analysed using the eBURST software program, which can be found at pubmlst.org/analysis/. The default eBURST setting identified groups of related STs using the most stringent (conservative) definition, such that all members assigned to the same group shared identical alleles at six of the seven loci with at least one other member of the group. The study STs were compared with all STs in the $S$. agalactiae MLST database. A phylogenetic tree was drawn from the allelic profile data using the Phylodendron software (pubmlst.org/analysis/), which employs an unweighted pair group method with an arithmetic averages (UPGMA) algorithm.

Sequencing of the modified glcK allele - In addition to the glcK amplification and sequencing primers from pubmlst.org/sagalactiae/, specific primers were designed to sequence the insert detected within the glcK amplicon of one strain using the Primo primer design tool (mobyle.pasteur.fr); the primers were based on the sequence of a previously published $g l c K$ allele available in GenBank (accession EF990366) (Martins et al. 2007). These primers are listed in Table I.

PFGE - The strains were compared by PFGE of SmaIdigested genomic DNA, using a protocol described elsewhere (Usein et al. 2012), on a CHEF-Mapper apparatus (Bio-Rad Laboratories, Hercules, CA, USA). Cluster analysis of the PFGE fragment patterns was performed using the Dice coefficient at $1.5 \%$ tolerance and $1.5 \%$ 
TABLE I

Primers designed in this study for sequencing the insert of $g l c K$ allele

\begin{tabular}{lc}
\hline Primer & $\begin{array}{c}\text { Nucleotide sequence } \\
\left(5^{\prime} \rightarrow 3^{\prime}\right)\end{array}$ \\
\hline glcK_362F & CAGGTGGAGAAATTGGGC \\
glcK_716F & GATGGCACCTAAGGTATTCAC \\
glcK_1079F & TCAGGATTGTCAAGGCTACTC \\
glcK_1518R & AGGAGAAGCTTTTCGTGATAG \\
glcK_1882R & TCAATTCTTTAGCCTCTAATGC \\
glcK_2236R & CCTTTCTTTGGATGACGACG \\
glcK_2769R & TGTCGCTGATGCAACTG \\
\hline
\end{tabular}

optimisation and a dendrogram was constructed using the UPGMA algorithm and the Bionumerics software (Applied Maths, Sint-Martens-Latem, Begium). The results were interpreted according to the criteria proposed by van Belkum et al. (2007). Strains with PFGE profiles that differed by four or fewer bands were assigned to subtypes of the same PFGE type (pulsotype) and isolates with patterns that differed by five or more bands were assigned to distinct types.

cps genotype - A previously published, 19-plex PCR assay for the identification of the Ia, Ib and II-IX GBS capsular types was used, with slight modifications (Imperi et al. 2010). PCRs were performed in a final volume of $25 \mu \mathrm{L}$. Each PCR reaction mixture contained 5 $\mu \mathrm{L}$ of bacterial lysate, 1x Qiagen Multiplex PCR Master Mix (Qiagen, Hilden, Germany) and the 19-primer pool (primers 2-15, 17-19 at 6 pmoles each and primers 1 and 16 at 10 pmoles each). Amplification was carried out in a GeneAmp PCR System 2700 (Applied Biosystems) and the following thermocycling conditions were used: an initial denaturation of $95^{\circ} \mathrm{C}$ for $15 \mathrm{~min}$ (to activate HotStarTaq DNA polymerase) followed by 15 cycles of $94^{\circ} \mathrm{C}$ for $30 \mathrm{~s}, 54^{\circ} \mathrm{C}$ for $90 \mathrm{~s}$ and $72^{\circ} \mathrm{C}$ for $90 \mathrm{~s}$, then 25 cycles of $94^{\circ} \mathrm{C}$ for $30 \mathrm{~s}, 56^{\circ} \mathrm{C}$ for $90 \mathrm{~s}$ and $72^{\circ} \mathrm{C}$ for $90 \mathrm{~s}$ and a final extension at $72^{\circ} \mathrm{C}$ for $10 \mathrm{~min}$.

Molecular traits - Surface protein coding gene profile and MGE detection - Previously described primers and protocols were used to identify the presence of the bac, bca, rib, alp1, alp $2 / 3$ and alp 4 genes that encode $\mathrm{C}$ beta, $\mathrm{C}$ alpha, Rib and the alpha-like proteins Alp1, Alp2, Alp3, Alp4, respectively, as well as the insertion of the MGEs IS1548 and GBSil within the $s c p B-\operatorname{lmb}$ genetic region (Usein et al. 2012).

Pilus-coding gene profile - Previously published primers were used to screen for the presence of PI-1, PI2a and PI-2b (Margarit et al. 2009). The PI-1 backbone protein-coding gene (1,798 bp expected amplicon), the PI-2a ancillary protein-coding gene $(\sim 2,840$ bp expected amplicon) and the PI-2b backbone-coding gene (1,700 bp expected amplicon) were selected as targets for the identification of the PI-1, PI-2a and PI-2b-like regions.
The PCRs had a final reaction volume of $50 \mu \mathrm{L}$, which contained $5 \mu \mathrm{L}$ of bacterial lysate, 1x High Fidelity PCR Master (Roche, Mannheim, Germany) and 15 pmoles each of the forward and reverse primers. The PCRs were run on a GeneAmp PCR System 2700 (Applied Biosystems), using the following thermal cycling conditions: an initial denaturation of $94^{\circ} \mathrm{C}$ for $3 \mathrm{~min}$ followed by 9 cycles of $94^{\circ} \mathrm{C}$ for $20 \mathrm{~s}, 55^{\circ} \mathrm{C}$ for $60 \mathrm{~s}$ and $72^{\circ} \mathrm{C}$ for $90 \mathrm{~s}$, then 24 cycles of $94^{\circ} \mathrm{C}$ for $20 \mathrm{~s}, 55^{\circ} \mathrm{C}$ for $30 \mathrm{~s}$ and $72^{\circ} \mathrm{C}$ for $90 \mathrm{~s}$ plus $5 \mathrm{~s}$ for each successive cycle and a final extension at $72^{\circ} \mathrm{C}$ for $7 \mathrm{~min}$.

\section{RESULTS}

MLST analysis - The 55 strains examined in this study were resolved into 18 unique STs. The number of allelic profiles for each of the seven housekeeping genes of the GBS MLST scheme ranged from three (pheS) to 11 alleles (atr). We identified four atr alleles (atr-63, atr64, atr-65 and atr-66) and five STs (ST-587 and ST-592 through 595), which were newly assigned by the database curator. Four of them were variants related to ST-19 (ST-594, ST-595), ST-22 (ST-592) and ST-297 (ST-593) with new atr alleles. ST-587 had a new combination of previously known alleles. The sequences of all the new alleles and the composition of the newly identified STs are available from pubmlst.org/sagalactiae/.

MLST genotyping revealed that six STs were represented by at least three strains each: ST-1 (8 strains), ST-8 (3 strains), ST-17 (13 strains), ST-19 (9 strains), ST-23 (3 strains) and ST-28 (6 strains). ST-297 was detected twice and 11 STs (ST-10, ST-12, ST-26, ST-49, ST-103, ST-335, ST-578 and ST-592 through ST-595) occurred only once. The presumed ST-17 status of the four strains from the previous study was confirmed by MLST.

The Romanian strains' MLST profiles were included in four of the 12 groups defined by eBURST when compared with all known allelic profiles from the GBS MLST database, downloaded on 11 July 2013 (Fig. 1). Fifteen STs (ST-1, ST-8, ST-10, ST-12, ST-17, ST-19, ST28, ST-49, ST-297, ST-335, ST-587 and ST-592 through ST-595) were categorised into group 1, the largest and most complex eBURST group. These STs clustered into multiple clonal complexes (CCs): CC19 (ST-19, ST-28, ST-335, ST-587, ST-594 and ST-595), CC1 (ST-1, ST-49, ST-297 and ST-593), CC10 (ST-10, ST-8 and ST-12), CC17 (ST-17) and CC67 (ST-592). The remaining three STs (ST-23, ST-103 and ST-26) were distributed into groups 2, 3 and 6, respectively, representing CC23, CC26 and CC103. When the autochthonous strains were analysed as an independent group, setting the group definition of seven shared alleles to zero, ST-17, ST-23, ST-26, ST-103 and ST-592 appeared as individual, unlinked STs (singletons), along with three clusters corresponding to the above CC19, CC1 and CC10 groups.

Sequence of the modified glcK allele - For one of the strains, the internal fragment of the $g l c K$ allele gave a larger-than-expected PCR product, estimated at approximately $3 \mathrm{~kb}$ instead of the predicted $0.459 \mathrm{~kb}$. DNA sequencing of the amplicon revealed that the increase in band size was due to a 2,313 bp insertion between nu- 


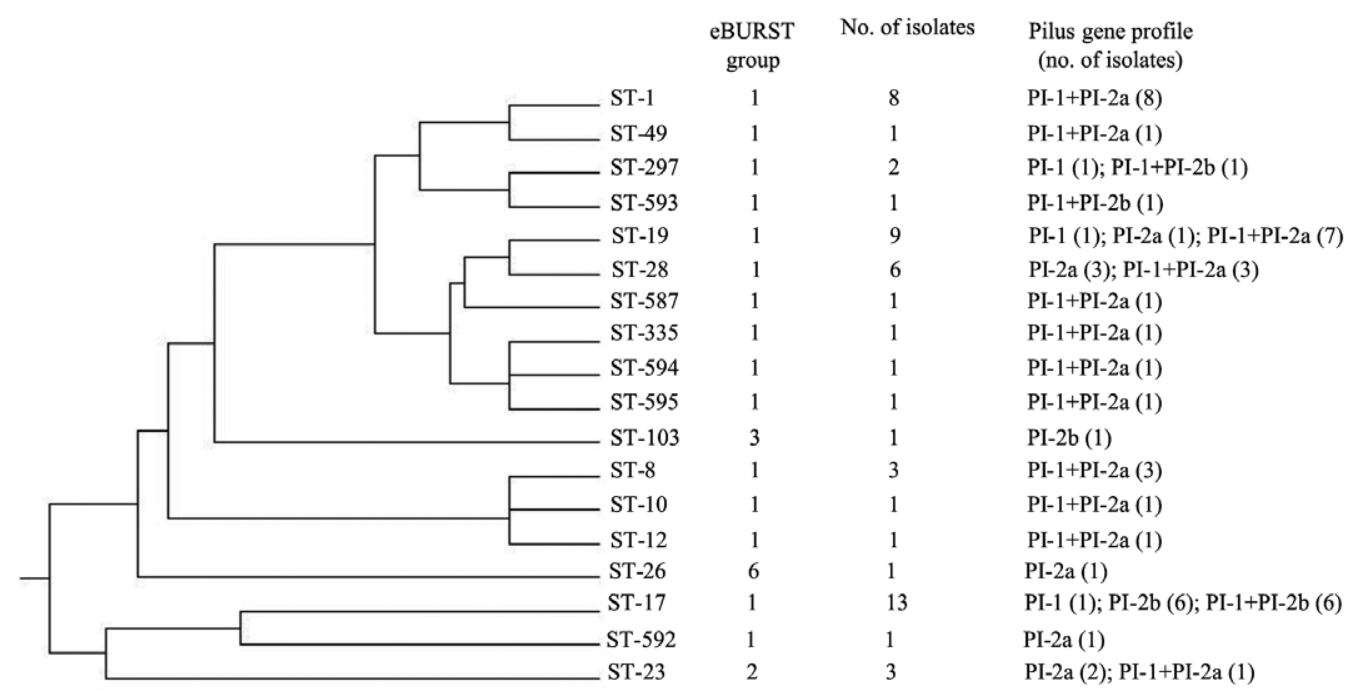

Fig. 1: unweighted pair group method with an arithmetic averages tree generated from the multilocus sequence typing allelic profiles of the 55 isolates in relation to the eBURST groups and pilus islands distribution.

cleotides 405 and 406 of the $g k c K$ fragment. A BLAST search identified an open reading frame (ORF) within this sequence, with $98 \%$ identity to a group II intron reverse transcriptase. The newly determined nucleotide sequence was deposited in GenBank (accession HF679144). The incorporated sequence was removed for MLST analysis, leaving an intact $g l c K$ fragment identified as allele 2. The complete allelic profile assigned the isolate to ST-1.

Presence of pilus islands and correlations with STs Genes associated with at least one of the three GBS pilus islands were detected in each of the 55 strains investigated. More precisely, PI-1 was detected in 40 strains, PI-2a in 37 strains and PI-2b in 15 strains. Nineteen strains displayed only one type of pilus island and 36 strains carried a two-pilus island combination. Consequently, the PCR results were considered predictive for the presence of the following profiles: PI-1 (3 strains), PI-2a (8 strains), PI-2b (7 strains), PI-1+PI-2a (29 strains) and PI$1+\mathrm{PI}-2 \mathrm{~b}$ (8 strains). The concurrent presence of PI-2a and PI- $2 \mathrm{~b}$ was not detected. For the STs represented by more than three strains, certain correlations were observed between the presence of a particular pilus island profile and the ST status. ST-1 strains exclusively displayed PI1+PI-2a profiles. With the exception of an ST-19 strain that carried PI-1 alone, the ST-19 and ST-28 strains contained PI-2a alone or in association with PI-1. All ST17 strains but one possessed PI-2b and this island was detected in only three other STs, namely ST-103, ST-297 and its single locus variant (SLV), ST-593.

In terms of the pilus island distribution across the serotypes, the PI-1+PI-2a profile was present in strains displaying cps genotypes $\mathrm{V}$ (17 strains), III (5 strains), II (5 strains) and $\mathrm{Ib}$ ( 2 strains). PI-2b, with or without PI-1, was identified in cps III (12 strains), IV (2 strains) and Ia (1 strain).

Identification of cps genotypes, surface protein encoding genes and MGEs - Molecular serotyping success- fully assigned all 43 strains uncovered in this study to six cps types: Ia (3 strains), Ib (1 strain), II (7 strains), III (13 strains), IV (2 strains) and V (17 strains).

PCR amplification of the surface protein encoding genes revealed that 41 strains were positive for one of the following genes: $b c a$ (4 strains), alpl (12 strains), alp $2 / 3$ (7 strains) and rib (18 strains). Three of the $b c a-$ positive strains concurrently carried the $C \beta$ protein gene, bac. Two strains were PCR-negative for all of the surface protein-encoding genes investigated. Twenty-nine strains $(67 \%)$ harboured either the group II intron GBSi1 (20 strains) or the insertion sequence IS1548 (9 strains) within the $s c p B$ - lmb genetic region.

The 12 strains that were obtained from a previous study were assigned to the following cps types: type $\mathrm{Ib}$ (1 strain), type II (2 strains), type III (5 strains), type IV (1 strain) and type V (3 strains). These strains harboured the following surface protein encoding genes: bca (1 strain), alp1 (2 strains) alp2/3 (2 strains) rib (7 strains) and $b a c$ (1 strain). The $b c a$ and $b a c$ genes were associated. Four of the 12 strains contained neither of the two examined MGEs, whereas seven were positive for the intron GBSi1 and one carried IS1548.

Table II presents the relationships between the sequence types, cps genotypes and the combinations of various genetic markers (surface protein genes, MGEs, pilus islands) for all 55 strains.

Antimicrobial resistance profiles, resistance genotypes and associations with cps type - All 43 isolates were susceptible to penicillin, but resistant to tetracycline either due to the presence of tet $M$ alone (35 strains) or due to tet $M$ in association with tet $O$ (8 strains). Erythromycin resistance was observed in 19 strains. Nine of these strains displayed a constitutive clindamycin resistance phenotype due to $\operatorname{erm} B$, while 10 strains expressed an inducible phenotype due to ermA alone (5 strains), 
TABLE II

Relationships between sequence types, capsular polysaccharide synthesis (cps) genotypes and the molecular marker profiles (i.e. surface protein genes, mobile genetic elements, pilus islands) for the 55 studied strains

\begin{tabular}{lcc}
\hline & $c p s$ genotype & Molecular marker profile \\
Sequence & (isolates) & (isolates) \\
type & (n) & (n)
\end{tabular}

\begin{tabular}{|c|c|c|}
\hline 1 & $\mathrm{~V}(8)$ & $\begin{array}{c}a l p 2 / 3+\text { PI-1, PI-2a (7) } \\
r i b+\text { PI-1, PI-2a (1) }\end{array}$ \\
\hline \multirow[t]{2}{*}{8} & $\mathrm{Ib}(1)$ & $b a c, b c a+$ PI-1, PI-2a (1) \\
\hline & $\mathrm{V}(2)$ & $b a c, b c a+$ GBSil + PI-1,PI-2a (2) \\
\hline 10 & II (1) & alp1 + GBSi1 + PI-1, PI-2a (1) \\
\hline 12 & $\mathrm{Ib}(1)$ & $b a c, b c a+\mathrm{PI}-1, \mathrm{PI}-2 \mathrm{a}(1)$ \\
\hline \multirow[t]{3}{*}{17} & III (13) & $r i b+$ GBSi1 + PI-1, PI-2b (6) \\
\hline & & $r i b+$ GBSi1 + PI-2b (6) \\
\hline & & $r i b+$ GBSi1 + PI-1 (1) \\
\hline \multirow[t]{5}{*}{19} & III (2) & rib + IS1548 + PI-1, PI-2a (2) \\
\hline & $\mathrm{V}(7)$ & alp1 + IS1548 + PI-1, PI-2a (3) \\
\hline & & alp1 + GBSi1 + PI-1, PI-2a (2) \\
\hline & & alp1 + IS1548 + PI-1 (1) \\
\hline & & alp1 + IS1548 + PI-2a (1) \\
\hline \multirow[t]{2}{*}{23} & Ia (2) & alp1 + PI-2a (2) \\
\hline & III (1) & alp2/3 + PI-1, PI-2a (1) \\
\hline 26 & V (1) & GBSi1 + PI-2a (1) \\
\hline \multirow[t]{2}{*}{28} & II (6) & $r i b+$ GBSi1 + PI-1, PI-2a (3) \\
\hline & & $r i b+$ GBSi1 + PI-2a (3) \\
\hline 49 & $\mathrm{~V}(1)$ & alp2/3 + PI-1, PI-2a (1) \\
\hline 103 & Ia (1) & PI-2b (1) \\
\hline \multirow[t]{2}{*}{297} & IV (2) & alpl + PI-1, PI-2b (1) \\
\hline & & alp1 + PI-1 (1) \\
\hline 335 & III (1) & $r i b+\mathrm{IS} 1548+\mathrm{PI}-1, \mathrm{PI}-2 \mathrm{a}(1)$ \\
\hline 578 & II (1) & $r i b+$ GBSi1 + PI-1, PI-2a (1) \\
\hline 592 & II (1) & $b c a+$ IS1548 + PI-2a (1) \\
\hline 593 & IV (1) & alp1 + PI-1, PI-2b (1) \\
\hline 594 & III (1) & rib + IS1548 + PI-1, PI-2a (1) \\
\hline 595 & $\mathrm{~V}(1)$ & alp1 + GBSi1 + PI-1, PI-2a (1) \\
\hline
\end{tabular}

erm $A$ associated with mefA (4 strains) or ermB (1 strain). Five of the strains with inducible resistance to clindamycin were concurrently resistant to levofloxacin and moxifloxacin (Fig. 2). These strains displayed the cps $\mathrm{V}$ genotype and the parC and gyrA sequences, including the QRDRs responsible for fluoroquinolone (FQ) resistance, showed an identical pattern of nucleotide mutations. Specifically, the strains presented single substitutions in GyrA (Ser81Leu) and ParC (Ser79Phe), as well as identical silent mutations that were found exclusively in $\operatorname{parC}$ (codons 142 and 145).

PFGE analysis of FQ-resistant (FQR) isolates - FQR strains were also subjected to PFGE analysis. All strains yielded interpretable PFGE profiles following SmaI digestion and these profiles were compared using an UPGMA-based dendrogram (Fig. 2). The FQR cluster extended to $72 \%$ similarity, corresponding to a total of four DNA fragments or bands that differed. Consequently, these strains were assigned to the same pulsotype according to the recommendations of van Belkum et al. (2007). Four subtypes were identified within this pulsotype, with $86 \%$ similarity and two bands that differed. Two strains were considered indistinguishable and were assigned to the same subtype.

\section{DISCUSSION}

This is the first Romanian study to investigate the diversity of the GBS strains colonising the human female population using the MLST approach linked to the analysis of significant genetic markers for GBS description. This comprehensive genotyping system generated a highly informative characterisation of autochthonous strains and allowed for objective comparisons with strains from other geographic regions. Overall, the GBS population sampled in this study comprised 18 ST profiles, six capsular genotypes and 20 molecular profiles defined by the combination of three sets of markers ( 6 surface protein genes, 2 MGEs and 3 pilus islands).

Most STs identified in this study have been previously described; however, despite the small number of strains, we also identified five STs that were not present in the MLST database. The most prevalent STs in our data set were ST-1, ST-17, ST-19 and ST-28. These results were partially in agreement with a study of a diverse, globally derived GBS collection of 67 strains isolated from adult carriers (Jones et al. 2003). In that study, the ST-1, ST-17 and ST-19 strains also occurred frequently, but the ST-28 clone was less prevalent. By contrast, a higher proportion of ST-23 members was reported, a finding that was also reported in a study of colonising strains isolated from young nonpregnant American women (Manning et al. 2008). In the latter study, ST-22 was the second-most prevalent clone identified after ST-1, followed by ST-23 and ST-19. ST-22, a clone that also frequently occurred in pregnant women from Israel (Bisharat et al. 2005), was not identified among the Romanian samples, except for one strain that was assigned to ST-592, an ST-22 SLV. ST-19 and ST-28 belong to the same CC19 group. ST28 strains were more prevalent within CC19 in a study focusing on strains isolated from maternal carriage in two different African cities, Dakar and Bangui (Brochet et al. 2009). In the Romanian collection, as well as in others from Europe and the United States, ST-19 was described as the most prevalent ST included in this CC.

The ST-17 clone, which is significantly associated with invasive infections, was strongly represented in the present study. A frequent occurrence of lineage ST-17 colonisation has also been reported for pregnant East African women, German adults and Jews and non-Jews who emigrated from the former Soviet republics (Marchaim et al. 2006, Eickel et al. 2009, Huber et al. 2011).

For STs with many isolates, such as ST-1, ST-17, ST23 and ST-28, we found several associations between the MLST profiles and the molecular markers screened in this study. Among this collection's strains, the cps II, III and V types predominated. In agreement with other studies, although a capsular serotype was generally not 


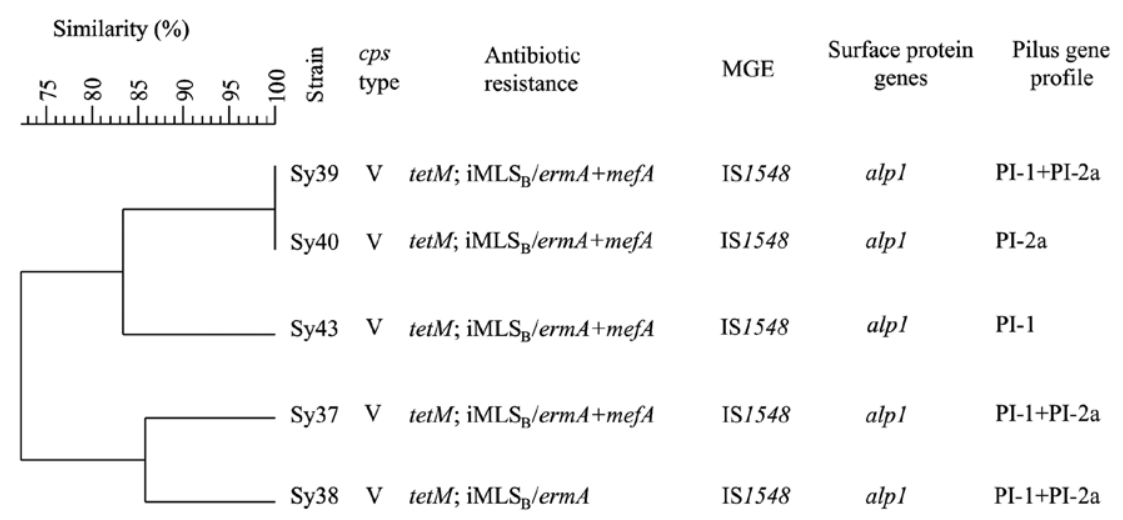

Fig. 2: characteristics of the fluoroquinolone-resistant strains assigned to sequence types-19 lineage in relation with the genetic relatedness of the strains as revealed by the unweighted pair group method with an arithmetic averages dendrogram of their SmaI pulsed-field gel electrophoresis profiles.

restricted to a specific ST, we observed the tendency for a single serotype to prevail within individual STs (Brochet et al. 2006, Bohnsack et al. 2008). In this study, the exclusive associations ST-1/cps V, ST-17/cps III and ST28/cps II should be mentioned. In contrast with other studies that reported a strong association of ST-19 with serotype III, in our collection, the members of this clone were more frequently associated with serotype V (Marchaim et al. 2006, Manning et al. 2009).

Also of note, one of the ST-1/cps V strains in this study carried a putative group II intron inserted within the $g l c K$ internal fragment, which was discovered in the GBS MLST scheme. While searching the literature for similar findings, we found three other studies that mentioned isolates harbouring the same type of insertion, each located exclusively in a $g l c K$ allele in the same position (Bisharat et al. 2004, Brochet et al. 2006, Martins et al. 2007); however, the sequence data that were available for comparisons were limited. The previously described isolates were mainly of bovine origin and belonged to other lineages (e.g., ST-61). The only reported strain of human origin belonged to serotype Ia and the ST-248 lineage (Brochet et al. 2006).

Correlations were observed between the cps types and the surface protein genes. The cps III and II type strains were closely associated with $r i b$ and the cps $\mathrm{Ib}$ strain was associated with $b a c$ and $b c a$, which was consistent with other reports (Kong et al. 2002, Martins et al. 2011). The association of serotype $\mathrm{V}$ with alp 3 that was evident in other studies (Kong et al. 2002, Martins et al. 2011) was not encountered in our collection, where a similar number of strains displaying the cps $\mathrm{V}$ genotype harboured either alp2/3 or alp1.

Previous studies revealed some associations between certain STs and surface protein genes. In addition to the expected ST-17/rib association, we observed that the ST28/cps II Romanian strains also exclusively harboured $r i b$. In contrast, the ST-28 strains of serotype II from a German colonising collection carried either $b c a$ or alp2/3 (Eickel et al. 2009). Two serotypes were detected in the ST-8 strains, but both serotypes carried the same association with $b a c$ and $b c a$. This was not the case for ST-19 and ST-23, both of which included serotypes that har- boured different surface protein encoding genes. Interestingly, the only representative of ST-26 from our collection lacked surface protein genes, which was similar to results found in a Polish study (Sadowy et al. 2010).

Al Safadi et al. (2010) reported that most CC1 strains did not harbour either GBSil or IS1548 within the $s c p B$ lmb intergenic region and mainly found GBSil in CC17 strains of serotype III and CC19 strains of serotype II. Our results showed both similarities to and differences from that study. We also noticed that the $\mathrm{CCl}$ isolates (ST-1, ST-49, ST-297 and ST-593) lacked MGEs, whereas all of the ST-17 isolates exclusively contained the GBSil intron. Within the CC19 cluster, the ST-28/cps II strains displayed GBSil, whereas most ST-19 isolates (78\%) harboured IS1548. The preponderance of the ST19/IS1548 isolates with the cps V genotype was mainly due to a group of isolates with genetic homogeneity, according to PFGE typing, that acquired resistance to FQs. These strains were resistant to tetracycline and macrolides, with an iMLS $_{\mathrm{B}}$ phenotype correlated with the presence of ermA and mefA. Double and even triple combinations of erm and/or mefA have been reported by other authors (Culebras et al. 2002, Fluegge et al. 2004), but these combinations were not found in our Romanian GBS isolates. FQ resistance was supported by key mutations detected in the QRDRs of gyrA and parC, which encode the GyrA and ParC subunits of the DNA gyrase and topoisomerase IV, respectively. The inferred alterations of these enzymes seemed to be sufficient to cause clinical resistance to levofloxacin and moxifloxacin (Tazi et al. 2008). The assumption of a spread of multidrug resistant GBS isolates based on our study would be misguided, due to the small number of isolates studied; however, the presence of a predominant pulsotype might be indicative of the local circulation of genetically related FQR isolates. Based only on the results of PFGE analysis, a Japanese study concluded that FQR isolates, mainly of serotype $\mathrm{Ib}$, might belong to a single clone that acquired FQ resistance and rapidly disseminated throughout Japan (Murayama et al. 2009). Further, a recent report from China revealed that a large percentage of FQR GBS was due to serotype III isolates of the ST-19 lineage (Wang et al. 2013). 
In this study, for the first time, we screened Romanian GBS strains for the presence of the genetic determinants of pilus-like structures, which are considered promising vaccine candidates (Margarit et al. 2009). All of the studied strains carried at least one pilus island and the most common pilus island pattern consisted of the concomitant presence of the PI-1 and PI-2a loci, as previously reported for colonising strains from Europe or the United States of America (Margarit et al. 2009). Across our collection, this combination was identified in more than half of the studied strains (53\%) belonging to $12 \mathrm{STs}$. For prevalent STs, such as ST-1 and ST-19, the PI-1+PI2a genotype prevailed. In a recent study, Martins et al. (2013) noted the dominance of the PI-1+PI-2a combination in several worldwide-disseminated lineages, as well as its nearly ubiquitous presence in isolates belonging to $\mathrm{CC} 19$ and its association with maternal colonisation. While $69 \%$ of the local strains carried PI-2a alone or in combination with PI-1, the PI- $2 \mathrm{~b}$ and PI-1+PI-2b profiles were detected in fewer isolates (27\%), mainly in those of the serotype III and ST-17 lineage. The significant association of PI-2b, alone or in combination with PI-1, with this particular lineage has been previously reported (Madzivhandila et al. 2013) and it was suggested that the acquisition of this variant might be partly responsible for this lineage's particular virulence and tropism (Martins et al. 2013).

The data acquired in this study shed new light on the need for a more rigorous characterisation of the GBS isolates circulating in our area and on the value of expanding the laboratory typing toolset by including methods such as MLST, which are helpful for both local and global GBS epidemiology.

\section{REFERENCES}

Al Safadi R, Amor S, Hery-Arnaud G, Spellerberg B, Lanotte P, Mereghetti L, Gannier F, Quentin R, Rosenau A 2010. Enhanced expression of $l m b$ gene encoding laminin-binding protein in Streptococcus agalactiae strains harboring IS1548 in scpB-lmb intergenic region. PLoS ONE 5: e10794.

Bellais S, Six A, Fouet A, Longo M, Dmytruk N, Glaser P, Trieu-Cuot P, Poyart C 2012. Capsular switching in group B streptococcus CC17 hypervirulent clone: a future challenge for polysaccharide vaccine development. $J$ Infect Dis 206: 1745-1752.

Bisharat N, Crook DW, Leigh J, Harding RM, Ward PN, Coffey TJ, Maiden MC, Peto T, Jones N 2004. Hyperinvasive neonatal group B streptococcus has arisen from a bovine ancestor. J Clin Microbiol 42: 2161-2167.

Bisharat N, Jones N, Marchaim D, Block C, Harding RM, Yagupsky P, Peto T, Crook DW 2005. Population structure of group B streptococcus from a low-incidence region for invasive neonatal disease. Microbiology 151: 1875-1881.

Bohnsack JF, Whiting A, Gottschalk M, Dunn DM, Weiss R, Azimi PH, Philips JB 3rd, Weisman LE, Rhoads GG, Lin FY 2008. Population structure of invasive and colonizing strains of Streptococcus agalactiae from neonates of six US academic centers from 1995 to 1999. J Clin Microbiol 46: 1285-1291.

Brochet M, Couvé E, Bercion R, Sire JM, Glaser P 2009. Population structure of human isolates of Streptococcus agalactiae from Dakar and Bangui. J Clin Microbiol 47: 800-803.

Brochet M, Couvé E, Zouine M, Vallaeys T, Rusniok C, Lamy M-C, Buchrieser C, Trieu-Cuot P, Kunst F, Poyart C, Glaser P 2006.
Genomic diversity and evolution within the species Streptococcus agalactiae. Microbes Infect 8: 1227-1243.

Castor ML, Whitney CG, Como-Sabetti K, Facklam RR, Ferrieri P, Bartkus JM, Juni BA, Cieslak PR, Farley MM, Dumas NB, Schrag SJ, Lynfield R 2008. Antibiotic resistance patterns in invasive group B streptococcal isolates. Infect Dis Obstet Gynecol 2008: ID727505.

Culebras E, Rodriguez-Avial I, Betriu C, Redondo M, Picazo JJ 2002. Macrolide and tetracycline resistance and molecular relationships of clinical strains of Streptococcus agalactiae. Antimicrob Agents Chemother 46: 1574-1576.

Diedrick MJ, Flores AE, Hillier SL, Creti R, Ferrieri P 2010. Clonal analysis of colonizing group B Streptococcus, serotype IV, an emerging pathogen in the United States. J Clin Microbiol 48: 3100-3104.

Edwards MS, Baker KJ 2005. Group B streptococcal infections in elderly adults. Clin Infect Dis 41: 839-847.

Eickel V, Kahl B, Reinisch B, Dübbers A, Küster P, Brandt C, Spellerberg B 2009. Emergence of respiratory Streptococcus agalactiae isolates in cystic fibrosis patients. PLOS ONE 4: e4650.

Fasola E, Livdahl C, Ferrieri P 1993. Molecular analysis of multiple isolates of the major serotypes of group B streptococci. J Clin Microbiol 31: 2616-2620.

Fluegge K, Supper S, Siedler A, Berner R 2004. Antibiotic susceptibility in neonatal invasive isolates of Streptococcus agalactiae in a 2-year nationwide surveillance study in Germany. Antimicrob Agents Chemother 48: 4444-4446.

Huber CA, McOdimba F, Pflueger V, Daubenberger CA, Revathi G 2011. Characterization of invasive and colonizing isolates of Streptococcus agalactiae in East African adults. J Clin Microbiol 49: 3652-3655.

Imperi M, Pataracchia M, Alfarone G, Baldassarri L, Orefici G, Creti R 2010. A multiplex PCR assay for the direct identification of the capsular type (Ia to IX) of Streptococcus agalactiae. J Microbiol Methods 80: 212-214.

Jones N, Bohnsack JF, Takahashi S, Oliver KA, Chan MS, Kunst F, Glaser P, Rusniok C, Crook DW, Harding RM, Bisharat N, Spratt BG 2003. Multilocus sequence typing system for group B streptococcus. J Clin Microbiol 41: 2530-2536.

Kawamura Y, Fujiwara H, Mishima N, Tanaka Y, Tanimoto A, Ikawa S, Itoh Y, Ezaki T 2003. First Streptococcus agalactiae isolates highly resistant to quinolones, with point mutations in gyrA and parC. Antimicrob Agents Chemother 47: 3605-3609.

Kong F, Gowan S, Martin D, James G, Gilbert GL 2002. Molecular profiles of group B streptococcal surface protein antigen genes: relationship to molecular serotypes. J Clin Microbiol 40: 620-626.

Lamy MC, Dramsi S, Billoët A, Réglier-Poupet H, Tazi A, Raymond J, Guérin F, Couvé E, Kunst F, Glaser P, Trieu-Cuot P, Poyart C 2006. Rapid detection of the "highly virulent" group B Streptococcus ST-17 clone. Microbes Infect 8: 1714-1722.

Lauer P, Rinaudo CD, Soriani M, Margarit I, Maione D, Rosini R, Taddei AR, Mora M, Rappuoli R, Grandi G, Telford JL 2005. Genome analysis reveals pili in Group B Streptococcus. Science 309: 105.

Madzivhandila M, Adrian PV, Cutland CL, Kuwanda L, Madhi SA, PoPS Trial Team 2013. Distribution of pilus islands of group B streptococcus associated with maternal colonization and invasive disease in South Africa. J Med Microbiol 62: 249-253.

Manning SD, Schaeffer KE, Springman AC, Lehotzky E, Lewis MA, Ouellette LM, Wu G, Moorer GM, Whittam TS, Davies HD 2008. Genetic diversity and antimicrobial resistance in group B streptococcus colonizing young, nonpregnant women. Clin Infect Dis 47: 388-390. 
Manning SD, Springman AC, Lehotzky E, Lewis MA, Whittam TS, Davies HD 2009. Multilocus sequence types associated with neonatal group B streptococcal sepsis and meningitis in Canada. J Clin Microbiol 47: 1143-1148.

Marchaim D, Efrati S, Melamed R, Gortzak-Uzan L, Riesenberg K, Zaidenstein R, Schlaeffer F 2006. Clonal variability of group B Streptococcus among different groups of carriers in southern Israel. Eur J Clin Microbiol Infect Dis 25: 443-448.

Margarit I, Rinaudo CD, Galeotti CL, Maione D, Ghezzo C, Buttazzoni E, Rosini R, Runci Y, Mora M, Buccato S, Pagani M, Tresoldi E, Berardi A, Creti R, Baker CJ, Telford JL, Grandi G 2009. Preventing bacterial infections with pilus-based vaccines: the group B streptococcus paradigm. Infect Dis 199: 108-115.

Martins ER, Andreu A, Correia P, Juncosa T, Bosch J, Ramirez, MeloCristino J 2011. Group B streptococci causing neonatal infections in Barcelona are a stable clonal population: 18-year surveillance. J Clin Microbiol 49: 2911-2918.

Martins ER, Andreu A, Melo-Cristino J, Ramirez M 2013. Distribution of pilus islands in Streptococcus agalactiae that cause human infections: insights into evolution and implication for vaccine development. Clin Vaccine Immunol 20: 313-316.

Martins ER, Melo-Cristino J, Ramirez M 2007. Reevaluating the serotype II capsular locus of Streptococcus agalactiae. J Clin Microbiol 45: 3384-3386.

Murayama SY, Seki C, Sakata H, Sunaoshi K, Nakayama E, Iwata S, Sunakawa K, Ubukata K, Invasive Streptococcal Disease Working Group 2009. Capsular type and antibiotic resistance in Streptococcus agalactiae isolates from patients, ranging from newborns to the elderly, with invasive infections. Antimicrob Agents Chemother 53: 2650-2653.
Sadowy E, Matynia B, Hryniewicz W 2010. Population structure, virulence factors and resistance determinants of invasive, noninvasive and colonizing Streptococcus agalactiae in Poland. J Antimicrob Chemother 65: 1907-1914.

Slotved HC, Kong F, Lambertsen L, Sauer S, Gilbert GL 2007. Serotype IX, a proposed new Streptococcus agalactiae serotype. J Clin Microbiol 45: 2929-2936.

Tazi A, Gueudet T, Varon E, Gilly L, Trieu-Cuot P, Poyart C 2008. Fluoroquinolone-resistant group B streptococci in acute exacerbation of chronic bronchitis. Emerg Infect Dis 14: 349-350.

Telford JL, Barocchi MA, Margarit I, Rappuoli R, Grandi G 2006. Pili in Gram-positive pathogens. Nat Rev Microbiol 4: 509-519.

Usein CR, Grigore L, Georgescu R, Cristea V, Bãltoiu M, Strãuț M 2012. Molecular characterization of adult-colonizing Streptococcus agalactiae from an area-based surveillance study in Romania. Eur J Clin Microbiol Infect Dis 31: 2301-2310.

van Belkum A, Tassios PT, Dijkshoorn L, Haeggman S, Cookson B, Fry NK, Fussing V, Green J, Feil E, Gerner-Smidt P, Brisse S, Struelens M, European Society of Clinical Microbiology and Infectious Diseases (ESCMID), Study Group on Epidemiological Markers (ESGEM) 2007. Guidelines for the validation and application of typing methods for use in bacterial epidemiology. Clin Microbiol Infect 13: 1-46.

Wang H, Zhao C, He W, Zhang F, Zhang L, Cao B, Sun Z, Xu Y, Yang Q, Mei Y, Hu B, Chu Y, Liao K, Yu Y, Hu Z, Ni Y 2013. High prevalence of fluoroquinolone-resistant group B streptococci among clinical isolates in China and predominance of sequence type 19 with serotype III. Antimicrob Agents Chemother 57: 1538-1541. 